ORGANISATIONAL MATTERS

\title{
A case of the birth and death of a high reliability healthcare organisation
}

\author{
K H Roberts, P Madsen, V Desai, D Van Stralen
}

Qual Saf Health Care 2005;14:216-220. doi: 10.1136/qshc.2003.009589

High reliability organisations (HROs) are those in which errors rarely occur. To accomplish this they conduct relatively error free operations over long periods of time and make consistently good decisions resulting in high quality and reliability. Some organisational processes that characterise HROs are process auditing, implementing appropriate reward systems, avoiding quality degradation, appropriately perceiving that risk exists and developing strategies to deal with it, and command and control. Command and control processes include migrating decision making, redundancy in people or hardware, developing situational awareness, formal rules and procedures, and training. These processes must be tailored to the specific organisation implementing them. These processes were applied to a paediatric intensive care unit (PICU) where care was derived from problem solving methodology rather than protocol. After a leadership change, the unit returned to the hierarchical medical model of care. Important outcome variables such as infant mortality, patient return to the PICU after discharge, days on the PICU, air transports, degraded. Implications for clinical practice include providing caregivers with sufficient flexibility to meet changing situations, encouraging teamwork, and avoiding shaming, naming, and blaming.

See end of article for authors' affiliations

Correspondence to: Dr K H Roberts, Walter A Haas School of Business, 545 Student Services Building, University of California, Berkeley, CA 94720, USA; Karlene@ haas.berkeley.edu

Accepted for publication 12 April 2005
$\mathrm{H}$ igh reliability organisations (HROs) are organisations in which errors can have catastrophic consequences but which consistently seem to avoid such errors. To accomplish this they conduct relatively error-free operations over a long period of time, and make consistently good decisions, resulting in high quality and reliability. Examples of such organisations include many commercial nuclear power plants, commercial aviation, the operation of the US Navy's carrier aviation program, Kaiser Permanente's western region neonatal program, and the US Federal Aviation Administration's air traffic control system. These organisations rarely find themselves in trouble but, when they do, they engage in rigorous searches to ascertain what went wrong. ${ }^{1}$

The writer Robert Pool says in his book "Beyond Engineering: How Society Shapes Technology": ${ }^{2}$

"In a generation or two, the world will likely need thousands of high reliability organisations, running not just nuclear power plants, space flights, and air traffic control, but also chemical plants, electrical grids, computer and telecommunication networks, financial networks, genetic engineering, nuclear waste storage, and many other complex, hazardous technologies. Our ability to manage a technology, rather than our ability to conceive and build it, may be the limiting factor in many cases."

Here we describe the components of high reliability, present a case study of the processes involved in building an HRO and the benefits realised in doing so, and examine the reversal of these processes and their consequences. The causes of the reversal and lessons for other organisations are addressed.

While HROs have many unique characteristics including their functions, hierarchies, and complexities, they all engage in similar processes towards meeting their common goal of reliability enhancement.

\section{ELEMENTS OF A HIGH RELIABILITY ORGANISATION (HRO)}

In her research in financial institutions, Carolyn Libuser identified the following five elements of HROs (box 1): ${ }^{3}$

- Process auditing or an established system for ongoing checks designed to identify expected as well as unexpected safety problems. Safety drills and equipment testing fall into this category. Follow up of problems revealed in prior audits is critical.

- The reward system is the payoff an individual or organisation receives for behaving one way or another. Organisational theory shows that organisational reward systems have powerful influences on the behaviour of people in them. Similarly, inter-organisational reward systems influence behaviour in organisations.

- Avoiding degradation of quality and/or avoiding development of inferior quality. This refers to the essential quality of the system compared with the referent generally regarded as the standard of quality in the industry.

- Risk perception: (1) whether or not there is knowledge of risk and (2) if there is knowledge that risk exists, the extent to which it is acknowledged and appropriately mitigated. Part 2 is the logical outgrowth of part 1 .

- Command and control which consists of five elements:

Abbreviations: $\mathrm{HRO}$, high reliability organisation; $\mathrm{PICU}$, paediatric intensive care unit; $R C P$, respiratory care practitioner 


\section{Box 1 Elements of an HRO}

- Process auditing

- Appropriate reward system

- Avoiding quality degradation

- Risk perception

- Command and control

- Migrating decision making

- Redundancy of people or hardware

- Senior managers with "the big picture"

- Formal rules and procedures

- Training

- Migrating decision making: the person with the most expertise makes the decision regardless of where that person is in the hierarchy.

- Redundancy of people or hardware: this consists of some kind of back up system. Duplication does not count as redundancy because, if both redundant systems are exactly alike, both have a higher probability of failure than if the back ups are unalike but complementary.

- Situational awareness: senior managers have the "big picture". They do not micromanage. They trust their subordinates are well enough trained to do their jobs without micromanagement. There are many examples of catastrophic accidents because no one was overseeing the whole operation.

- Formal rules and procedures: a definite existence of hierarchy but not necessarily bureaucracy in the negative sense.

- Training: training is like the three rules of real estatelocation, location, location-but in this case it is training, training, and more training. And what is the first thing to go when organisations find themselves in a financial bind?

These processes have been applied in a number of organisations in various industries. That they have relevance to the healthcare industry is evident from their successful application to obstetric nursing at the University of Minnesota Medical School and Kaiser Permanente's western region neonatal services. These theoretical notions are the basis for the application described here.

Box 2 The PICU at Back Bay Children's Hospital (BBCH)

- Beds: 250

- Average daily census: 21

- Children on ventilators: 9

- Registered nurses: 105

- Nurses on duty at any one time: 14

- Respiratory care practitioners: 220

- Rotating resident physicians: 4

- Paediatric intensivists: 5

- Medical director is paediatric intensivist

\section{A HIGHLY RELIABLE HEALTHCARE UNIT}

\section{The setting}

Back Bay Children's Hospital (BBCH) has 250 beds and is the tertiary children's hospital for a geographical area more than three times the size of the state of Vermont (box 2). During the period that the paediatric intensive care unit (PICU) operated under the principles of HRO, 2.5 million people populated the locality with 500000 under the age of 15. The region includes urban, rural and wilderness areas, with a large number of desert and mountain communities.

Medical care was provided by the intensivist who saw each patient twice daily on rounds. Morning rounds were for teaching and the group consisted of all residents, the fellow if on service, lead respiratory care practitioner (RCP), charge nurse, pharmacist, social worker, and the patient's bedside nurse and respiratory therapist. Students from nursing, respiratory care, medicine, social work, and paramedic training colleges also accompanied the team. Afternoon rounds reviewed the day's progress, the patient's response to treatment, and discussed the plan for the night.

The PICU had 1704 admissions in 1996 making it one of the largest PICUs in the country, both in number of beds and admissions. Pollack and colleagues ${ }^{4}$ found mortality rates of $7.8(0.8) \%$ for PICUs with more than 18 beds. The PICU at $\mathrm{BBCH}$ had a $5.2 \%$ mortality rate in 1996 .

About half the admissions came through the paediatric critical care transport system at BBCH, now one of the larger transport services in the country. Most (75\%) paediatric specialised transport systems carry less than 400 patients per year, with the largest transporting 720 patients. The year of the McCloskey study, ${ }^{5} \mathrm{BBCH}$ paediatric critical care transport brought 599 children to the PICU. This did not include neonates transported to the neonatal intensive care unit. In $1996 \mathrm{BBCH}$ transported 871 children to that unit.

\section{HRO as process}

Before $\mathrm{BBCH}$ adopted $\mathrm{HRO}$ processes, children transported to it had about twice the mortality rate of those admitted from within the institution (via emergency department, operating room, or acute care ward). $\mathrm{McNab}^{6}$ found post-transport mortality of $11.5 \%$ when he reviewed 130 charts of children with a decreased level of consciousness. BBCH had one deterioration due to cardiac arrest in 1991 which did not result in death. In the spring of 1993 three children died during transport in a 6 month period.

Following these three problems, the transport medical director changed the approach towards moribund children being transported from distant referral facilities (mostly emergency departments). Any child with the potential for airway instability or deterioration would receive endotracheal intubation before leaving the emergency department. Rather than using blood gas measurements and chest radiographs, a clinical five-point respiratory examination was developed. ${ }^{7}$ After implementation of this program and while high reliability efforts were in place, no child deteriorated during transport. In 1996 there were no adverse events during transport.

The PICU had more long term employees (more than 5 years) than other ICUs at Back Bay Medical Center (BBMC) or BBCH. Attrition was about 5\%, which was lower than adult ICUs. Institutional stress was lower than in other units, although there tended to be greater stress from the nature of the patients cared for in the PICU. When nurses left it tended to be for personal reasons or for academic and/or professional advancement.

\section{Philosophy of the PICU}

The philosophy of the PICU was to support the bedside caregiver, particularly the nurse, in an environment that had 
numerous social and psychological hazards. Teamwork and goal directed team formation were fostered rather than team formation by status and role. Shaming, naming and blaming-particularly after a bad outcome-were not accepted. There were many ways to approach care in the PICU; no one method was touted above the rest. Care was derived from problem solving methodology rather than protocol, policy, or algorithm. This allowed various services to pursue their own unique philosophies.

The centre of care was the team and support for the team leader and bedside caregiver. Objectives were developed for each patient and problem. Freedom to try interventions to identify what would work for a particular patient was allowed. Attending physician support was always available, either immediately by telephone or within 20 minutes in person. Because blame and guilt were not used, caregivers felt free to discuss problems early in their course. Deficiencies in care were used as teaching opportunities as every caregiver was valued as a long term member of the team.

\section{APPLICATION OF HRO ELEMENTS TO BBCH PICU}

Each organisation must apply the basic tenets of HROs in whatever way it sees as best. This is how the BBCH PICU adopted those tenets.

\section{Process auditing}

The process provided critical care medicine in an environment of physiological uncertainty and instability. Staff constantly entertained the thought that they missed something. They encouraged questioning and the presentation of data that supported or refuted their working hypotheses. Any team member could question care at any time. Questions were dealt with through education of all members. This led to immediate changes in patient care.

\section{Appropriate reward system}

Appropriate awards were given to encourage participation in patient care. These were often intrinsic. Any team member may have had information (percipient or opinion) that led to a solution. As members demonstrated knowledge, insight and discretion in patient care, they tended to play a greater role in tactical and strategic management. Their opinions were more frequently sought and incorporated into care plans. Through participation of all disciplines, the team sought to lower accidents and stress levels and improve morale of the caregivers.

\section{Avoiding quality degradation}

Quality review was performed to ensure the PICU had the lowest rate of potentially preventable mortality and morbidity. When possible, this review occurred during or as soon after an event as possible. Close involvement of attending physicians improved information flow between bedside caregivers and attending physicians. Quality improvement reviews were prepared. When an event produced consequential injury, the administration (medical centre and medical staff) became involved. This was after the fact, but with the intent of using such a situation as a marker of deficiency that needed to be reviewed. Quality referent levels for quality improvement were adopted from nationally accepted norms and the medical, respiratory care, and nursing literature.

\section{Risk perception}

Risk awareness was a more significant problem early in the growth of the PICU. As the team brought some control over the deranged physiology of critical illness or injury, the child often appeared physiologically quiet. Some caregivers mistook this for a recovering state rather than one of latent danger. Risk awareness remained an issue as the PICU introduced new high risk treatments.

Risk awareness increased over the first several years and the goal was to identify a child in the covert state of compensated physiological dysfunction. To do this the PICU began a program of in-service lectures specific to the various disciplines (nursing, respiratory care, resident physicians) and specific to the child and disease process. These bedside lectures occurred at all hours, lasting long enough for the staff to feel comfortable providing care. The staff also developed two regularly scheduled conferences, one directed at emergency medical service providers and the other directed at nurses in emergency departments and intensive care units. By 1997 it was the exception for a patient to deteriorate on the hospital ward, and it was also a rarity for a patient to unexpectedly deteriorate in the PICU. Few if any patients were re-admitted to the PICU after discharge.

\section{Command and control}

Command and control played a major part of care and gave the PICU its greatest successes. Decisions migrated to the best qualified team member. At the interface with the patient emergency, the most qualified person to make or guide decisions is the bedside caregiver. Frequently, caregivers could not predict what would work in a specific situation. They observed responses to treatment and often quickly made decisions which brought stability to rapidly changing situations. Responses to treatment guided further treatment and helped make the diagnosis. In that sense, there was no wrong decision as any decision and its action generated knowledge that could be used in treating the patient.

The authority gradient that frequently occurs between the physician or surgeon and other team members can lead to tragedy. There were times when particular services received bad news by returning anger, indifference, or failure to respond. To ameliorate this, nursing staff made greater use of a form for professional interactions. These forms went up the chain of command from the nurse to administration. They then moved downward to the physician involved through his/ her chain of command. This insulated nurses from reprisal. Anger and intimidation-tools used to maintain the authority gradient-became seen as a form of fight response of the familiar "fight or flight" fear reaction. People learned how to avoid using these tools.

Redundancy ensured thoroughness in evaluating the patient and in choosing a treatment. Many of the signs caregivers monitored were measured by two methods and during resuscitations several team members monitored the same vital sign.

Rules and procedures allowed RCPs and nurses to influence the medical care to a greater degree and with a quicker response to changes. Therapist-driven protocols allowed RCPs to take into consideration the patient's past response and expected response while deciding on treatment.

As a teaching institution and one that develops new treatments, the PICU at $\mathrm{BBCH}$ had the goal of always considering themselves in training. Consequently, they watched each other's performance and gave assistance through mutual teaching and learning.

\section{For 11 years $\mathrm{BBCH}$ was an $\mathrm{HRO}$}

During the 11 years the PICU operated as an HRO, in addition to the improvements discussed above, a number of other indicators of success changed in the right direction. Over that time period admissions went up as did the daily census and the use of ventilators. Mortality and consequential events went down. Transportation went up and refused transport requests went down. The unit was clearly a successful HRO. 


\section{THE DEMISE OF THE PICU}

After 11 years of successful operation, the two attending physicians who advocated for HRO left the PICU within a year of each other. They were replaced by several more intensive care attending physicians bringing the attending staff to six with two fellows. The service expanded to assist cardiothoracic surgeons in ICU care and a sedation service was developed.

None of the remaining staff had experience in other HROs outside medicine. Several had worked in this PICU while it was an HRO. They reverted to a medical model with the physician as leader, team by status and role, following of protocols and algorithms for safety, and maintaining central authority by the physician. Evidence-based medicine became the basis for treatment of critically ill unstable patients. These physicians considered the previous HRO model of decision migration unsafe. Loop decision making techniques to identify what works through action-for example, the Boyd OODA loop (Observe, Orient, Decide, Act)—were rejected as unknown and confusing. ${ }^{8}$

RCPs could no longer suggest treatments-in fact, some were criticized for making any suggestions. Attending staff provided more assistance through telephone consultations and distant evaluation. Bedside staff felt unsupported with unstable or deteriorating patients. Refusing ICU transfer requests confused physicians in the hospital and community who had become accustomed to the previous HRO model and concept that "the indication for PICU admission is the request by a physician". Patients discharged from the PICU were beginning to be readmitted within 48 hours.

Initially, only the physician culture changed from HRO to the standard medical model. Actions by nurses and RCPs continued but were beginning to be met with resistance, sometimes strongly worded. RCP attrition increased. New graduates began staffing the ICU-for example, while previous RCPs needed I year of ICU experience to transfer into the PICU, new graduates were now being taken.

What made this change of service acceptable? Why was it not identified? Because it follows the medical model so prevalent in health care and taught in healthcare training. People accepted as appropriate what they had always done. The medical model uses the physician as team leader and the team consists of positions determined by status and role. The physician evaluates the pertinent findings and comes to a conclusion-the diagnosis. The physician then generates a management plan. The more specific the diagnosis, the more specific the treatment. This is thought to increase safety and allow for more accurate risk-benefit assessment. The plan follows a logical progression of evaluation-treatment-reevaluation-more specific treatment. Such progressions easily fit into open tree decision schemata or algorithms. Central

\section{Key messages}

- High reliability organisations reduce errors and other negative outcomes.

- High reliability processes are easy to understand.

- Application of high reliability processes resulted in improved patient outcomes in a PICU.

- Implementation of high reliability processes require constant attention.

- Evidence based medical model of care does not work in situations where the patient's condition is rapidly deteriorating.

\section{Implications for clinical practice}

- If healthcare organisations are serious about reducing negative outcomes, they need to consider adopting high reliability processes.

- These processes can easily fail.

- They are costly in time, effort, and energy, but the cost is worth it when the organisation avoids unwanted outcomes.

authority is maintained by prior approval of protocols or by waiting for consultation with the treating physician.

This perspective does not allow flexible decisions based on inadequate, uncertain, or ambiguous data. It is inconsistent with the frequent time constraints required in a situation of rapidly evolving deterioration in the condition of the patient. We can see here that the medical model does not fit emergency medical situations, is in many respects diametrically opposite to the HRO model, and can contribute to patient mortality.

\section{MANAGING HROs FOR SAFE OPERATIONS}

As evidenced by several reports from the Institute of Medicine, safety in the US healthcare industry needs close attention. ${ }^{9}$ It should be clear to managers in the industry that, in situations in which conditions rapidly change, the evidence-based medical model of how to diagnose and manage does not work and is harmful. Department heads and their staffs need to develop true teamwork based on the problems they are likely to confront instead of the roles and status of team members. The unrecognised problem here is that often existing evidence is not very helpful, particularly in situations of tight time constraints. Caregivers often learn by doing, and they must be given sufficient flexibility to do so. They need to assess their units and organisations to make sure HRO processes are in place and that, when they need to be used, the people using them will not be punished. Teams may need to practice in simulations of the difficult problems that can come their way. Practice builds familiarity with interpersonal styles and skill levels and helps develop the flexibility required in these situations.

Our example shows that the application of Libuser's five principles resulted in improved care in a PICU. Each organisation must tailor its application to its own needs and maintain constant vigilance on reliability.

As evidenced from our example, HROs can easily turn into LROs (low reliability organisations). Thus, constant attention needs to be given to making sure the reliability glue is always in place. As we look at avoidable health related catastrophes, it seems the cost of avoiding failure is worth every penny.

\section{Authors' affiliations \\ K H Roberts, P Madsen, V Desai, Walter A Haas School of Business, University of California, Berkeley, CA, USA \\ D Van Stralen, Loma Linda University Medical School, Loma Linda, CA,} USA

This work was partially supported by National Science Foundation Grant SES-0105402.

\section{REFERENCES}

1 Roberts KH. Some characteristics of one type of high reliability organization. Organ Sci 1990;1:160-76.

2 Pool R. Beyond engineering: how society shapes technology. New York: Oxford University Press, 1997. 
3 Libuser CB. Organization structure and risk mitigation, Dissertation submitted in partial satisfaction of the requirements for the degree of Doctor of Philosophy in Management. Los Angeles: University of California, 1994.

4 Pollack MM, Cuerdon TC, Getson PR. Pediatric intensive care units: results of a national survey. Crit Care Med 1993;21:607-14.

5 McCloskey KA, Johnston C. Critical care interhospital transports: predictability of the need for a pediatrician. Pediatr Emerg Care 1990;6:89-92
$6 \mathrm{McNab}$ AJ. Optimal escort for interhospital transport of pediatric emergencies. J Trauma 1991;31:205-9.

7 Perkin RM, van Stralen DW. My child can't breathe: new tools for the recognition and early management of pediatric respiratory failure. J Emerg Med Serv 1993;24:43-56.

8 Coram R. Boyd: the fighter pilot who changed the art of war. Boston and New York: Little Brown, 2002:484

9 Kohn LT, Corrigan JM, Donaldson MS. To err is human: building a safer health system. Washington, DC: National Academy Press, 1999. 